\title{
Warning System for Abnormal Movement of Driver's Head
}

\author{
Jui-Cheng Yen*, Jia-Da Wang, Chong-Wei Li \\ Department of Electronic Engineering, National United University, Miao Li 360, Taiwan
}

*Corresponding Author: jcyen@ nuu.edu.tw

\begin{abstract}
The paper proposes a robust warning system to detect abnormal movement of driver's head. The LED light source is designed and placed behind the driver's head. The CMOS sensor is used to acquire frames including the region around the driver's head. The acquired frame is analyzed by the proposed abnormal head movement warning algorithm. Any head movement will result in changes of the LED region acquired by the CMOS sensor. By analyzing these changes, the monitoring algorithm can detect abnormal head movement and issue a warning signal correctly. The system is robust to violent illumination variation outside the vehicle and eye-sheltered effect from sunglasses. Software simulations are also given to demonstrate its effectiveness.
\end{abstract}

Keywords: safe driving, LED light source, CMOS sensor, illumination variation, sunglasses.

\section{Introduction}

When a driver is exhausted or absent-minded, his perception ability to surrounding environmental, handling ability to the car, and decision ability to traffic situation all diminish. They all easily result in serious traffic accident and loss of life and property. Hence, the development of safe driving system to detect whether a driver is exhausted is very important to prevent traffic accident. Actually different brands of car corporations and research centers devote themselves to develop this kind of system over the past decade ${ }^{(1-2)}$. For example, the monitoring system "City safety" is the standard equipment in VOLVO XC60.

Many approaches ${ }^{(2-11)}$ for safe driving have been proposed. They can be classified into the following four categories. 1) The first category ${ }^{(2-4)}$ is based on driver's physiological signals such as electrocardiogram signal, brain waves, and pulse rate. 2) The second category ${ }^{(2,5-7)}$ focuses on the state of driver's head or face such as head movement, blink rate, eye closure, eyelid movement, and pupillary variation. 3) The third category ${ }^{(2,8-9)}$ is based on vehicle's behavior such as vehicle lateral position in the lane, distance between the driver's vehicle and the front car, and movement direction. 4) Methods ${ }^{(2,10-11)}$ based on the combination of above categories are the fourth category.

In the first category, it is necessary to attach sensors to the driver to measure the physiological signal. The attachment will interfere with the driver and hence limit its practical application. On the contrary, to monitor the state of driver's head or face in the second category, it just needs non-contact CMOS sensors instead of contact ones. Well-developed image processing techniques make the second category to be a promising approach. However, how to overcome the violent illumination change outside the vehicle is a big challenge in image processing. Some techniques shine infrared rays on driver's eyes to produce bright effect on pupil of driver's eyes, use the infrared sensor to acquire the frame with bright pupil of driver's eyes, and analyze area change of pupil of driver's eyes to judge whether the driver is tired. The eyes can faithfully reflect whether the driver is exhausted and the pupil area can be adopted to estimate PERCLOS [6] to measure the driver's fatigue. However, this kind of system has three major disadvantages. 1) Shining infrared rays on driver's eyes continuously may be harmful to driver's eyes. 2) If the driver wears glasses, the light reflection from glasses will produce bright area which will be confused with the bright pupil. 3) The bright pupil effect is obvious in the nighttime, but it is unobvious in the daytime. Practical application is still a problem in such kind of system. Hence, the development of practical monitoring system safe to driver's eyes, robust to violent illumination variation, and not being effected by glasses is an important topic in the safe driving system.

In the paper, we propose a new system which preserves the advantages of the second category and improves its disadvantages. 


\section{Warning System for Abnormal Movement of Driver's Head}

\subsection{System Architecture}

To monitor whether the movement of the driver's head is abnormal, the warning system for abnormal movement of driver's head (WSAMDH) is proposed. Lateral and front views of the system are shown in Figs. 1(a) and 1(b) respectively. In the system, LED light source is adhered to the back cushion behind the driver's head. The CMOS sensor is used to acquire frames including the region around the driver's head. The acquired frame is analysed by the proposed abnormal head movement warning algorithm. Head movement causes driver's head covers different LED region and the LED region in the acquired frame changes. By analyzing these changes, the warning algorithm can detect abnormal head movement.

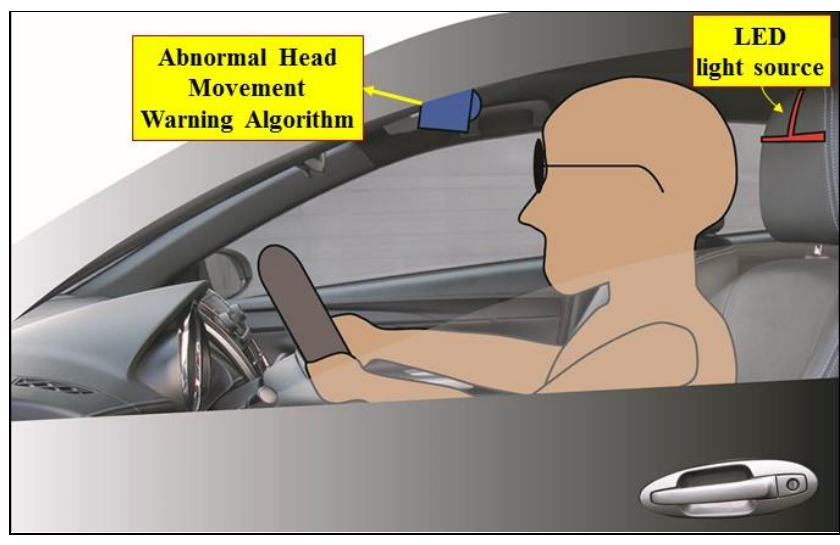

(a)

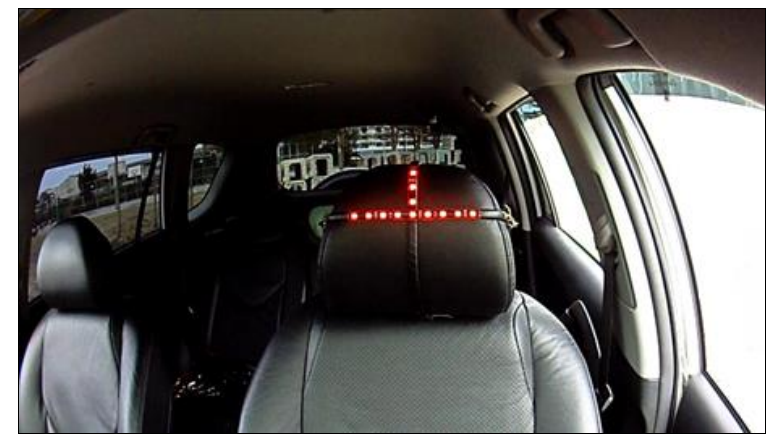

(b)

Fig. 1. WSAMDH (a) lateral view (b) front view.

\subsection{Abnormal Head Movement Warning Algorithm}

In WSAMDH, the abnormal head movement warning algorithm (AHMWA) shown in Figure 2 is proposed to monitor the changes of the LED region. To quantitatively describe AHMWA, the following notations are adopted and defined: $f d_{i}$ : the acquired $i$ th frame of size $540 \times 960$,

$f d \_H_{i}$ : horizontal region of interest (ROI) of size $17 \times 170$ in $f d_{i}$, $f d_{-} V_{i}$ : vertical ROI of size $50 \times 16$ in $f d_{i}$,

$f d \_H_{i}^{R}(p, q), f d \_H_{i}{ }^{G}(p, q)$, and $f d \_H_{i}{ }^{B}(p, q)$ : red, green, and blue components of $f d \_H_{i}(p, q), 1 \leq p \leq 17,1 \leq q \leq 170$,

$f d \_V_{i}^{R}(p, q), f d \_V_{i}^{G}(p, q)$, and $f d \_V_{i}^{B}(p, q)$ : red, green, and

blue components of $f d_{-} V_{i}(p, q), 1 \leq p \leq 50,1 \leq q \leq 16$,

$f d \_H S_{i}$ : binarization result from segmenting $f d \_H_{i}$,

$f d \_V S_{i}$ : binarization result from segmenting $f d \_V_{i}$,

area_HS $i$ : foreground area of $f d_{-} H S_{i}$,

area_VS : foreground area of $f d_{-} V S_{i}$,

ccog_HS : column coordinate of center of gravity (COG) of

$f d \_H S_{i}$,

warning $_{i}$ : indicator whether to issue a warning signal to the driver for frame $f d_{i}$.

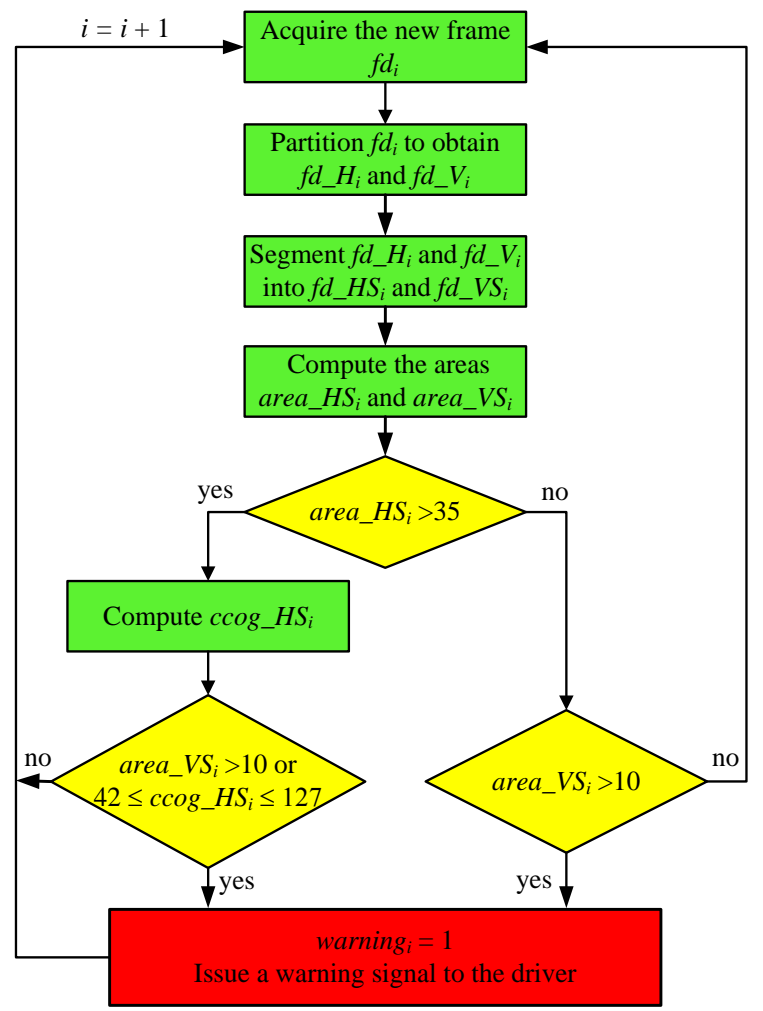

Fig. 2. The abnormal head movement warning algorithm in WSAMDH.

Based on the defined notations, AHMWA is proposed, shown in Figure 2, and described as follows.

Step 1: Acquire the new frame $f d_{i}, i=1,2,3, \ldots$

Step 2: Partition $f d_{i}$ to obtain $f d \_H_{i}$ and $f d \_V_{i}$

After acquiring $f d_{i}$, partition $f d_{i}$ to obtain $f d_{-} H_{i}$ and $f d_{-} V_{i}$. The two ROIs are defined as follows:

$$
\begin{aligned}
& f d \_H_{i}=\left\{f d_{i}(p, q) \mid 352 \leq p \leq 368,401 \leq q \leq 570\right\}, \\
& f d \_V_{i}=\left\{f d_{i}(p, q) \mid 303 \leq p \leq 352,478 \leq q \leq 493\right\} .
\end{aligned}
$$


Step 3: Segment $f d \_H_{i}$ and $f d \_V_{i}$ into $f d \_H S_{i}$ and $f d \_V S_{i}$

According to the color features of red, green, and blue components of $f d \_H_{i}$ and $f d \_V_{i}$, segment $f d \_H_{i}$ and $f d \_V_{i}$ by the following criteria:

$f d_{-} H S_{i}(p, q)= \begin{cases}1, & \text { if }\left|f d_{-} H_{i}^{R}(p, q)-f d_{-} H_{i}^{G}(p, q)\right|<25 \\ & \text { and } f d_{-} H_{i}^{B}(p, q)>200, \\ 0, & \text { otherwise, }\end{cases}$

$f d_{-} V S_{i}(r, s)= \begin{cases}1, & \text { if }\left|f d_{-} V_{i}^{R}(r, s)-f d_{-} V_{i}^{G}(r, s)\right|<25 \\ & \text { and } f d_{-} V_{i}^{B}(r, s)>200, \\ 0, & \text { otherwise, }\end{cases}$

where $1 \leq p \leq 17,1 \leq q \leq 170,1 \leq r \leq 50$, and $1 \leq s \leq 16$.

Step 4: Compute the areas $a r e a \_H S_{i}$ and $a r e a \_V S_{i}$

After segmenting $f d_{-} H_{i}$ and $f d_{-} V_{i}$, compute their foreground areas by the following methods:

$$
\begin{aligned}
& \text { area_HS }=\sum_{p=1}^{17} \sum_{q=1}^{170} f d_{-} H S_{i}(p, q), \\
& \text { area_VS }_{i}=\sum_{p=1}^{50} \sum_{q=1}^{16} f d_{-} V S_{i}(p, q) .
\end{aligned}
$$

Step 5: Determine whether the head movement is abnormal If area_HS $\leq 35$, inspect the following criterion:

$$
\text { Criterion 1: area_VS }>10 \text {. }
$$

If it is true, set warning $_{i}$ to be 1 and issue a warning signal to the driver.

If area_HS $>$ $>35$, compute the column coordinate of COG of $f d \_H S_{i}$ by

$$
c c o S_{-} H S_{i}= \begin{cases}0, & \text { if } \operatorname{area}_{-} H S_{i}=0, \\ \frac{\sum_{p=1}^{17} \sum_{q=1}^{170} q \times f d_{-} H S_{i}(p, q)}{\sum_{p=1}^{17} \sum_{q=1}^{170} f d_{-} H S_{i}(p, q)}, & \text { otherwise, }\end{cases}
$$

and inspect the following criteria:

$$
\text { Criterion 1: area_VS }>10 \text {, }
$$

Criterion 2: $42<c c o g \_H S_{i}<127$.

If either criterion is true, set warning $_{i}$ to be 1 and issue a warning signal to the driver.

In Step 2 of AHMWA, $f d \_H_{i}$ and $f d_{-} V_{i}$ of Fig. 1(b) are marked and shown in Fig. 3. $f d \_H_{i}$ and $f d \_V_{i}$ are regions of size $17 \times 170$ and $50 \times 16$ respectively. Both of them are small regions. Segmentation of ROIs by Eqs. (3)(4), computing areas of segmented ROIs by Eqs. (5)(6), and computing the column coordinate of COG of $f d_{-} H S_{i}$ by Eq. (7) are all simple operations and performed on the two small areas. Hence, the computational complexity of AHMWA is very low.

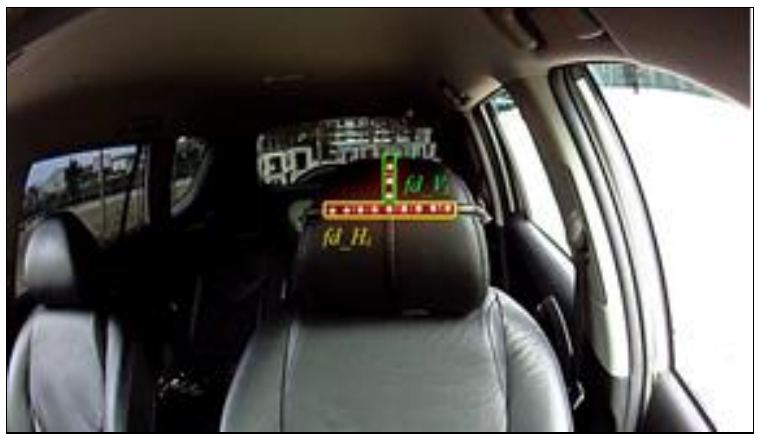

Fig. 3. $f d \_H_{i}$ and $f d \_V_{i}$.

If the drive's head is in the normal position, the LEDs in $f d \_H_{i}$ and $f d \_V_{i}$ are completely covered by driver's head. There is nothing in $f d \_H S_{i}$ and $f d_{-} V S_{i}$. The values of area_HS area_VS $S_{i}$, and $c c o g \_H S_{i}$ are all zero, hence the head movement is judged to be normal. If abnormal head movement occurs, the LEDs in $f d \_H_{i}$ and $f d \_V_{i}$ will be exposed. Hence, Step 5 can detect abnormal head movement and issue a warning signal to the driver.

\section{MATLAB Simulation Results}

To demonstrate the effectiveness of the proposed WSAMDH, the system is set up in an automobile. Two strips of SMD LED are adhered to the back cushion behind the driver's head. The vehicle video recorder CASA HDR-550 is used to record the region around the driver's head. The adopted video is of length about 59 seconds and includes the following two driving environments. 1) The driver wears the sunglasses. 2) The vehicle goes through the tunnel two times.

898 frames of size $540 \times 960$ in total are extracted from the video by the software "Free Video to JPG Converter". The acquisition rate is 15 frames per second. According to AHMWA, MATLAB simulation is performed on all the 898 frames. Use $f d_{510}$ as a representative to demonstrate the simulation results about ROI. $f d_{510}$ is shown in Fig. 4(a). The horizontal and vertical ROIs $f d_{-} H_{510}$ and $f d \_V_{510}$ are enlarged and shown in Figs. 4(b) and 4(c) respectively. Notice that $f d \_H_{510}$ and $f d \_V_{510}$ are regions of size $17 \times 170$ and $50 \times 16$ respectively. After segmenting $f d_{-} H_{510}$ and $f d \_V_{510}$ by Eqs. (3)(4), the results $f d \_H S_{510}$ and $f d \_V S_{510}$ are shown in Figs. 4(d) and 4(e) respectively. According to the execution results of AHMWA, the related values of $f d_{510}$ are listed in Table 1. From Table 1, we have area_HS $S_{510}=171$, 
area_VS $S_{510}=82$, and $c c o g \_H S_{510}=122$. Since area_HS $S_{510}$ is larger than 35 and both Criteria 1 and 2 are true, the head movement in $f d_{510}$ is judged to be abnormal. Hence, set warning $_{510}$ to be one and issue a warning signal to the driver.

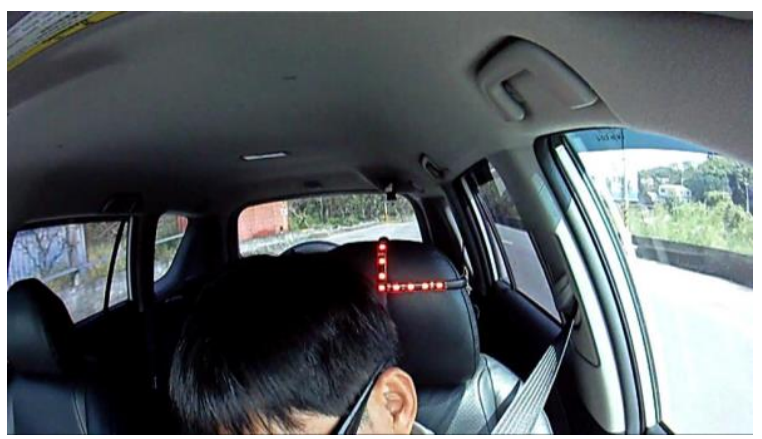

(a)

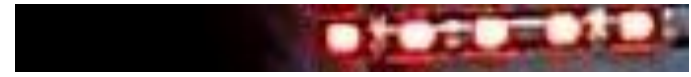

(b)

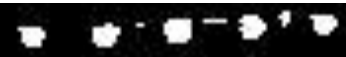

(d)

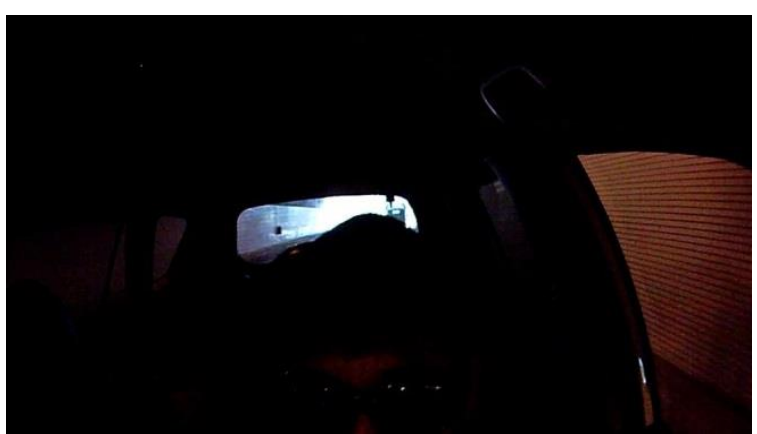

(a)

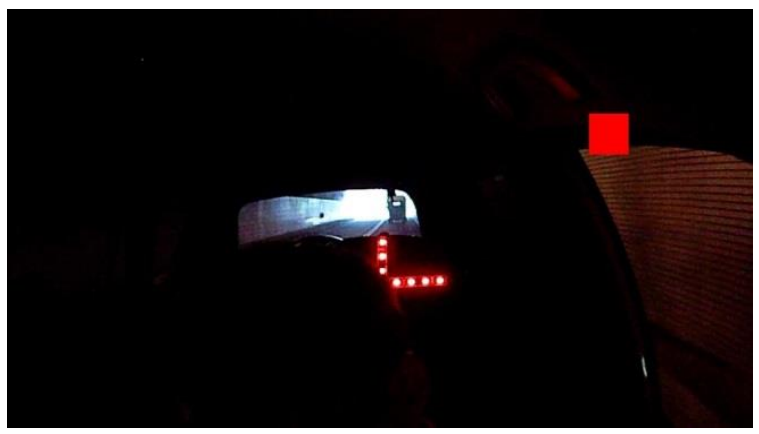

(b)

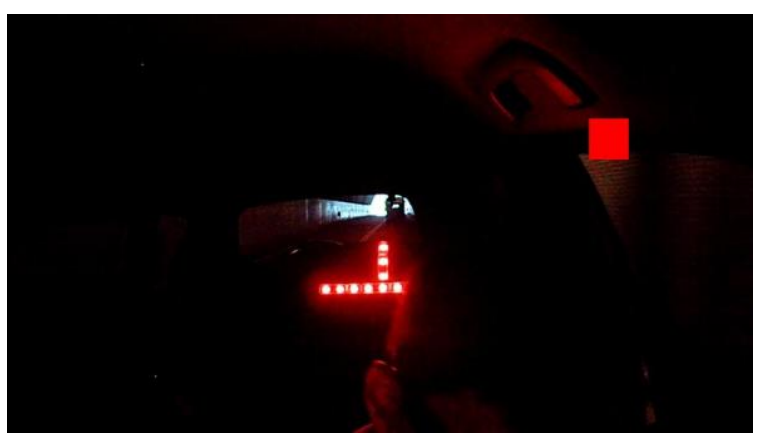

(c)

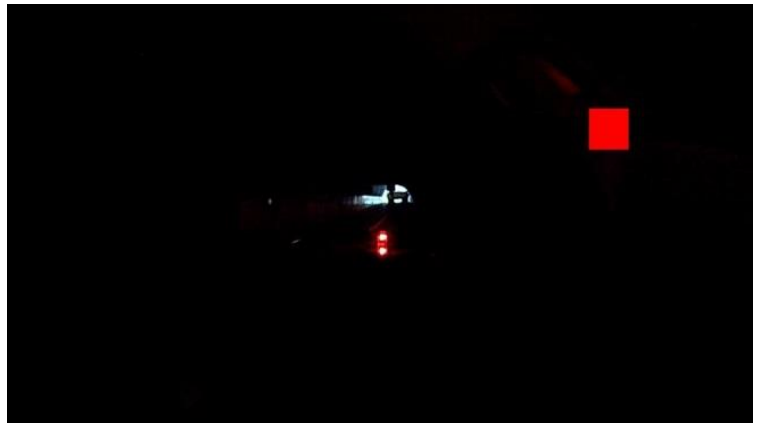

(d)

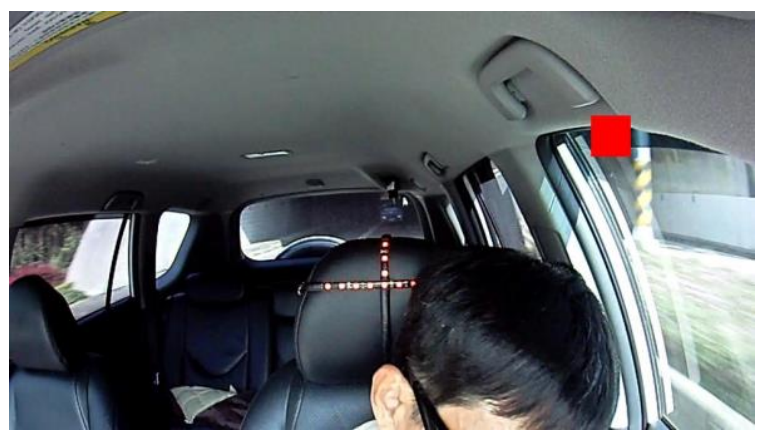

(e) 


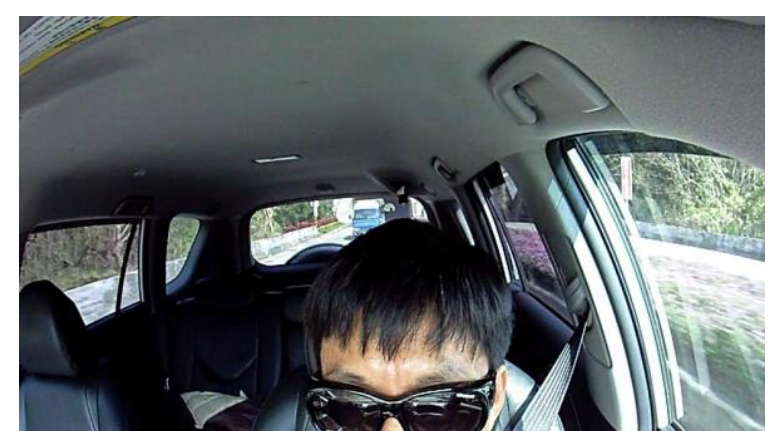

(f)

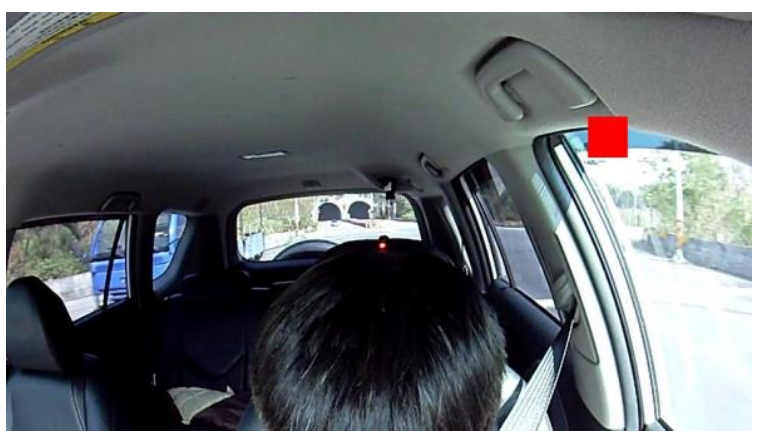

(g)

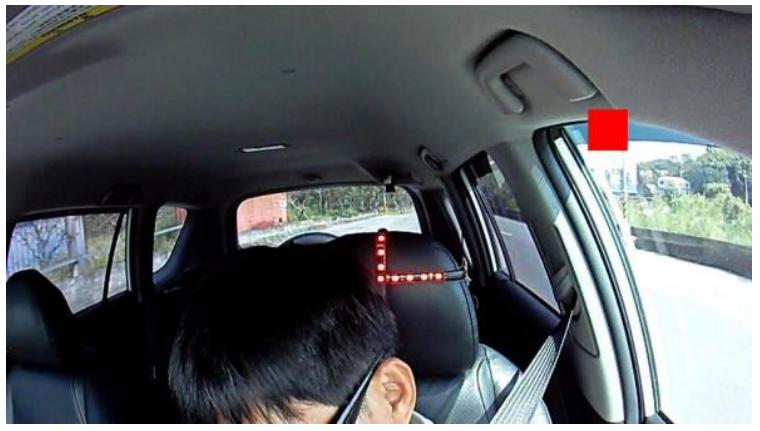

(h)

Fig. 5. The warning results of (a) $f d_{39}$, (b) $f d_{52}$, (c) $f d_{105}$, (d) $f d_{157}$, (e) $f d_{326}$, (f) $f d_{380}$, (g) $f d_{471}$, (h) $f d_{510}$.

Table 2. The related values of $f d_{i}$ according to AHMWA.

\begin{tabular}{|c|c|c|c|c|}
\hline \multirow{2}{*}{$\begin{array}{c}\text { Frame } \\
\text { no. } i\end{array}$} & \multicolumn{2}{|c|}{$f d_{-} H_{i}$} & $f d \_V_{i}$ & \multirow{2}{*}{ warning $_{i}$} \\
\cline { 2 - 4 } & area_HS & $c c o g_{-} H S_{i}$ & area_VS $_{i}$ & \\
\hline 39 & 0 & 0 & 0 & 0 \\
\hline 52 & 171 & 129 & 114 & 1 \\
\hline 105 & 424 & 60 & 206 & 1 \\
\hline 157 & 0 & 0 & 32 & 1 \\
\hline 326 & 261 & 60 & 69 & 1 \\
\hline 380 & 0 & 0 & 0 & 0 \\
\hline 471 & 0 & 0 & 14 & 1 \\
\hline 510 & 171 & 122 & 82 & 1 \\
\hline
\end{tabular}

For the simulation results of all the 898 frames, the warning indicators warning $_{i}, 1 \leq i \leq 898$, are shown in Fig. 6. Based on the warning indicators, we mark a red square on the corresponding frame. The complete simulation results of all the 898 frames are concatenated to a video again by the application software "Vegas Pro 8.0". The video is uploaded to YouTube ${ }^{(12)}$ and can also be played in Figure 7.

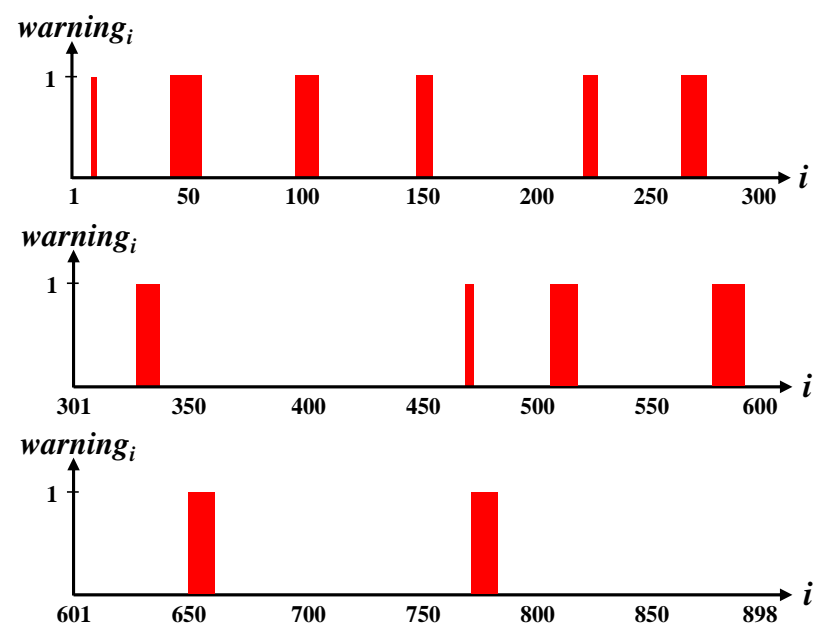

Fig. 6. The warning indicators of all the 898 frames

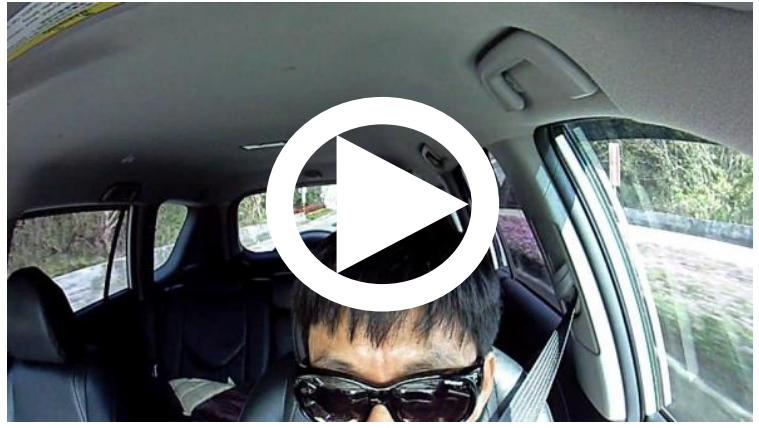

Fig. 7. The video of the simulation results uploaded to YouTube ${ }^{(12)}$.

In the acquired video, there are twelve times of driver's nods and two times of actions watching the rear-view of mirror. Inspecting the video demonstrated in Figure 7, all the nodding behaviors are detected exactly and the action the driver watches the rear-view mirror is judged to be a normal movement. The video also clearly demonstrates the features of the proposed WSAMDH. The driver wears the sunglasses and the automobile he drives goes through the tunnel two times. At the moment that the automobile enters or leaves the tunnel, the illumination variation outside the vehicle is very fiercely. Even so, WSAMDH overcomes the two challenges and detects the abnormal head movement correctly.

In the real system, the warning signal issued to the driver can be the sound from loudspeaker, figures in screen, 
music from player, light from source, vibration from vibrator, and so forth.

\section{Conclusions}

In the paper, the high-performance warning system for abnormal movement of driver's head has been proposed. The features of the proposed system are as follows. 1) The system can overcome the violent illumination change when the vehicle goes through the tunnel. 2) It is not influenced by sunglasses the driver wears. 3) The action the driver watches the rear-view mirror is judged to be a normal movement. 4) The AHMWA is a fast algorithm because only simple operations are performed on the two small areas. 5) It is a low-cost system.

Finally, we believe that the active safe driving system can greatly benefit by adopting the proposed system.

\section{Acknowledgment}

This work is supported in part by the Lien-Ho Foundation, R.O.C. under Grant 103-NUU-05.

\section{References}

(1) http://www.artc.org.tw/

(2) Y. Dong, Z. Hu, K. Uchimura, and N. Murayama, "Driver inattention monitoring system for intelligent vehicles: A Review," IEEE Trans. on Intelligent Transportation Systems, vol. 12, Iss. 2, pp. 596 - 614, June 2011.

(3) R. N. Khushaba, S. Kodagoda, S. Lal, and G. Dissanayake, "Driver drowsiness classification using fuzzy wavelet-packet-based feature-extraction algorithm," IEEE Trans. on Biomedical Eng., vol. 58, Iss. 1, pp. 121-131, Jan. 2011.

(4) A. Kircher, M. Uddman, and J. Sandin, "Vehicle control and drowsiness," Swedish National Road and Transport Research Institute, 2002.

(5) R. C. Coetzer, and G. P. Hancke, "Eye detection for a real-time vehicle driver fatigue monitoring system," 2011 IEEE Intelligent Vehicles Symp. (IV), pp. 66 - 71, 5-9 June 2011.

(6) D. F. Dinges, and R. Grace, "PERCLOS: A valid psychophysiological measure of alertness as assessed by psychomotor vigilance," US Department of Transportation, Federal highway Administration. Publication Number FHWA-MCRT-98-006.
(7) L. Li, Y. Chen and L. Xin, "Driver fatigue detection based on mouth information," 2010 8th World Congress on Intelligent Control and Auto. (WCICA), pp. 6058-6062, 7-9 July 2010

(8) C. F. Wu; C. J. Lin; C. Y. Lee, “Applying a functional neurofuzzy network to real-time lane detection and front-vehicle distance measurement," IEEE Trans. on Systems, Man, and Cybernetics, Part C: Applications and Reviews, vol. 42, Iss. 4, pp. 577-589, 2012.

(9) T. H. Chang, C. S. Hsu, C. Wang, L. K. Yang, "Onboard measurement and warning module for irregular vehicle behavior,” IEEE Trans. on Intelligent Transportation Systems, vol. 9, Iss. 3, pp. 501-513, 2008.

(10)B. G. Lee and W. Y. Chung, "Driver alertness monitoring using fusion of facial features and bio-signals," IEEE Sensors Journal, vol. 12 , pp. 2416-2422, 2012.

(11) B. G. Lee, S. J. Jung, and W. Y. Chung, "Real-time physiological and vision monitoring of vehicle driver for non-intrusive drowsiness detection," IET Communications, vol. 5, Iss. 17, pp. 2461-2469, 2011.

(12) https://www.youtube.com/watch?v=imW8DTs_23s\&fe ature=youtu.be 